\title{
Evaluación de las potencialidades turísticas de la parroquia rural Seis de Julio de Cuellaje
}

\section{Evaluation of the tourist potential of the rural community Seis de Julio de Cuellaje}

\author{
Lorena Elizabeth Casanova Imbaquingo ${ }^{1}$, Tatiana Elizabeth Guerrero Cabezas², \\ Juan Patricio Benítez Pereira ${ }^{3}$
}

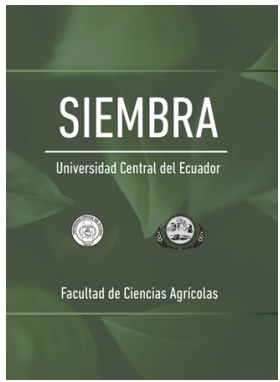

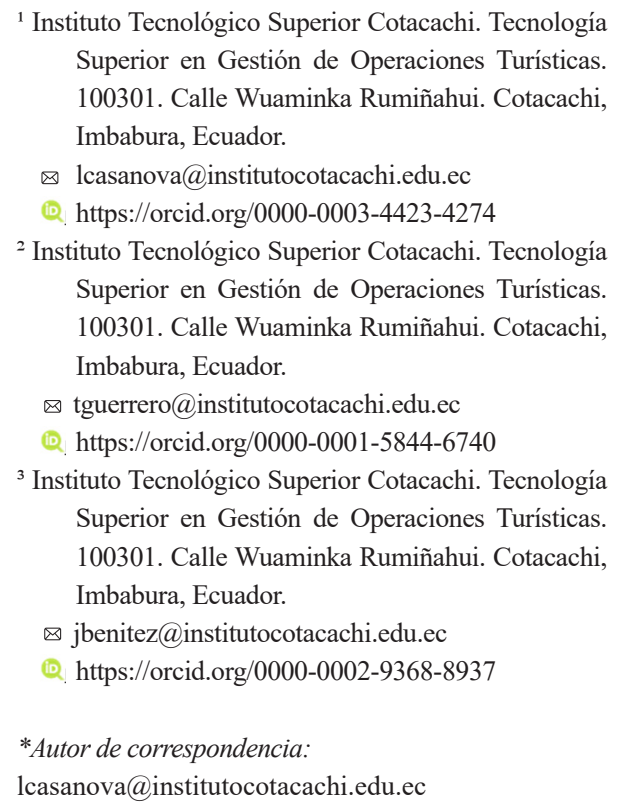

\section{SIEMBRA}

https://revistadigital.uce.edu.ec/index.php/SIEMBRA e-ISSN: $2477-8850$

\section{ISSN: $1390-8928$}

Periodicidad: semestral

vol. 9, núm. 1, 2022

siembra.fag@uce.edu.ec

DOI: https://doi.org/10.29166/siembra.v9i1.3420

\section{Resumen}

La situación económica a la que se enfrentan las parroquias rurales del Ecuador es preocupante debido a la escasa formulación de proyectos que contribuyan a crear oportunidades para mejorar las condiciones de vida de la población. En este sentido, el turismo puede constituirse en una alternativa de generación de empleo y crecimiento económico. El presente estudio tiene como objetivo determinar la potencialidad turística de la parroquia Seis de Julio de Cuellaje del cantón Cotacachi, provincia de Imbabura. Para el efecto, se empleó la metodología de jerarquización de atractivos y generación de espacios turísticos del Ministerio de Turismo del Ecuador 2018 y se adaptó la fórmula de evaluación multicriterio, misma que es igual a la división del criterio alcanzado por el atractivo sobre la ponderación estándar de los criterios de valoración. Se analizaron 20 atractivos turísticos, tres corresponden a la jerarquía II y 17 son de jerarquía I. Los criterios: estado de conservación e integración sitio/entorno, accesibilidad y conectividad obtuvieron mayor puntuación, sin embargo, su potencialidad es media. La puntuación de los siete criterios restantes es deficiente, por lo que se puede concluir que el nivel de potencialidad turística del territorio es bajo. Los actores públicos, privados y comunitarios deben trabajar mancomunadamente para mejorar las condiciones que actualmente desfavorecen al territorio como destino turístico emergente.

Palabras clave: atractivo turístico, criterios de valoración, nivel de potencialidad.
Abstract
The economic situation of rural cantons in Ecuador is disturbing due to the lack of formulation of projects that might contribute to create opportunities to improve living conditions of the population, being tour- ism an alternative for generating employment and economic growth. The objective of this study was to determine the tourist potential of the Seis de Julio de Cuellaje Cotacachi's canton, Imbabura province. For this purpose, the methodology of ranking attractions and generation of tourist spaces of Ministerio de Turismo del Ecuador was used and the general aptitude formula multicriterial was adapted, which is equal to the division of the criterion achieved by attractiveness over the standard 
weighting of endpoints. Twenty tourist attractions were analyzed, three correspond to hierarchy II and 17 are hierarchy I. The criteria: state of conservation and site/environment integration, accessibility and connectivity obtained higher scores: however, their potentiality is medium. The score of the seven remaining criteria is deficient, so it can be concluded that the level of tourist potential in the territory is low. Public, private and community actors must work together to improve the conditions that currently disadvantage the territory as an emerging tourist destination.

Keywords: tourist attraction, valuation criteria, level of potentiality.

\section{Introducción}

En el Ecuador, el turismo se ubica entre las principales actividades dinamizadoras de la economía y generadora de divisas (Segura Ronquillo, 2015). En el 2015 la contribución económica directa del turismo al Producto Interno Bruto (PIB) fue de USD 1.334.934 atribuidos directamente al consumo turístico interior; en el mismo año, el monto y participación del gasto turístico receptor alcanzó USD 1.851 .260 que constituye el 1,9\% del PIB (Ministerio de Turismo del Ecuador [MINTUR], 2021). La actividad turística influye en varios sectores económicos de forma directa o indirecta; así lo demuestran los encadenamientos de las actividades de alojamiento, alimentación y recreación; por ello, el turismo es considerado uno de los sectores más dinámicos y versátiles de las economías (Benavides Vindas, 2020). El MINTUR tiene como visión posicionar al Ecuador en la región como un destino turístico inclusivo, accesible, sostenible, competitivo e innovador (MINTUR, 2019).

La provincia de Imbabura fue declarada Geoparque Internacional por la Organización de las Naciones Unidas para la Educación, la Ciencia y la Cultura [UNESCO] en el 2018. La actividad turística en Imbabura representa el $3 \%$ del PIB provincial, porcentaje reflejado en los ingresos generados en establecimientos de alojamiento, alimentación y otros servicios asociados (Banco Central del Ecuador, 2017).

Imbabura tiene una amplia extensión geográfica con población rural significativa, diversidad étnica y riqueza de atractivos naturales y culturales no valorizados (Vasallo Villalonga et al., 2019). El desafío para Imbabura es esbozar los recursos naturales y culturales del territorio, aprovechando las externalidades que se ofrecen para el desarrollo de actividades turísticas; considerando que los principales motivos de viaje a la provincia son el ecoturismo y turismo de naturaleza (36\%), gastronomía (28\%) y atractivos religiosos-culturales (18\%) (Dávalos et al., 2019).

El turismo se constituye en una práctica eminentemente geográfica, siendo el territorio el escenario que acoge las experiencias turísticas (Garzón García y Florido Trujillo, 2021). El espacio geográfico debe presentar una serie de particularidades asociadas a los recursos naturales y culturales. Los recursos son considerados la materia prima que condiciona el potencial del destino, conjuntamente con otros servicios que dan respuesta a las necesidades de los visitantes (Camara et al., 2019).

Los elementos que complementan la materia prima del turismo comprenden las infraestructuras, equipamientos y servicios que conjugados estratégicamente dan paso a la generación de productos turísticos puestos a disposición del visitante (Camara et al., 2019). Los productos son consumidos in situ, ya que es el turista el que se traslada allí, donde se genera o produce la oferta, gracias a la atracción suscitada por los recursos vinculados al territorio (Garzón García y Florido Trujillo, 2021). El territorio turístico es, entonces, el escenario que integra recursos, infraestructuras y servicios que posibilitan la práctica turística.

El aprovechamiento de los recursos naturales y culturales del territorio para el desarrollo de actividades turísticas requiere la concertación público-privado, a nivel local/regional (Marzo-Navarro et al., 2017). La investigación determina que los esfuerzos invertidos y los recursos utilizados, tanto propios como externos al territorio, deben ser optimizados al máximo para la generación de productos turísticos con el propósito de promover la reactivación de la economía y la dinamización de una sociedad a través del turismo (Toselli, 2019).

La potencialidad turística es la relación existente entre los recursos -naturales y culturales-y su capacidad de satisfacer las necesidades del turista (Sánchez Zhingri et al., 2020). Las metodologías propuestas para determinar la potencialidad turística de un territorio comprenden, de manera general, el análisis de estas dos variables.

La potencialidad y aptitud del territorio se miden a través de la aplicación de una metodología basada en el análisis de los rasgos físicos, biológicos y atributos socioeconómicos que presenta una zona; cada dimensión aborda diferentes variables, seis de tipo natural y nueve socioeconómicas (Tabla 1). La información obtenida a través de entrevistas y fuentes institucionales se integra en un sistema de información geográfica y evaluación multicriterio (Nájera González et al., 2021). 
Tabla 1. Listado de variables analizadas.

Table 1. List of variables analyzed.

\begin{tabular}{ll}
\hline \multicolumn{1}{c}{ Dimensión } & \multicolumn{1}{c}{ Variables } \\
\hline \multirow{3}{*}{ Natural } & Unidades geomorfológicas \\
& El suelo \\
& La vegetación \\
& Hidrología \\
& Cercanía a poblaciones \\
& Curvas de nivel \\
\hline Semografía \\
Educación \\
Producción agropecuaria \\
Rasgos culturales \\
Servicios básicos \\
Infraestructura de accesibilidad \\
Infraestructura turística \\
Organización \\
Opinión sobre el turismo \\
\hline
\end{tabular}

Nota: tomado de «Proceso metodológico de evaluación de aptitud del territorio para actividades de turismo alternativo» (Nájera González et al., 2021).

La potencialidad de un territorio también se puede determinar con la aplicación de una metodología basada en el índice de potencialidad turística (IPT) compuesto por tres factores determinantes: los recursos de interés turístico, lo cuales a su vez se subdividen en naturales y culturales; la accesibilidad; y el equipamiento. El IPT es un cálculo que sirve para jerarquizar las localidades que poseen características con potencial para el desarrollo de actividades turísticas dentro de un área o zona (Flores Rodríguez et al., 2019).

En el Ecuador, el MINTUR, en el 2018, generó una Guía metodológica para la jerarquización de atractivos y generación de espacios turísticos, que tuvo como propósito la determinación del potencial turístico, basándose en nueve criterios de evaluación; seis criterios se relacionan con el índice de competitividad turística (accesibilidad y conectividad, planta turística y servicios, estado de conservación...) y tres están articulados a la demanda (difusión del atractivos, tipo de visitante y recurso humano).

La literatura demuestra la existencia de diversas metodologías que conllevan a la evaluación del potencial turístico de un territorio las cuales se apoyan en el análisis multicriterio de los recursos, la accesibilidad y el equipamiento (Sánchez Zhingri et al., 2020). La determinación del potencial turístico del territorio es un análisis útil de reconocimiento y zonificación de los recursos (naturales, sociales y económicos) a fin de buscar el aprovechamiento sustentable del territorio (Nájera González et al., 2021).

El objetivo principal de esta investigación es evaluar la potencialidad turística de la parroquia Seis de Julio de Cuellaje del cantón Cotacachi. El estudio se realiza aplicando la metodología para jerarquización de atractivos y generación de espacios turísticos, propuesta por el MINTUR (2018). Para medir la potencialidad se usó como principio la fórmula general de aptitud (Bravo Peña et al., 2015) la cual expresa que la potencialidad es igual a la sumatoria del peso del factor o criterio por el valor del factor o criterio. En este caso se empleó el peso del criterio determinado en la metodología del MINTUR. Los hallazgos de la investigación servirán como insumo para la consolidación de la planificación turística local.

\section{Materiales y métodos}

\section{1. Área de estudio}

El presente estudio se realizó en la parroquia Seis de Julio de Cuellaje, localizada en el cantón Cotacachi de la provincia de Imbabura-Ecuador. La parroquia está conformada por 8 comunidades: El Rosario, San Antonio, Playa Rica, Nápoles, Magdalena, San Joaquín, San Alberto y La Loma. Forma parte del Parque 


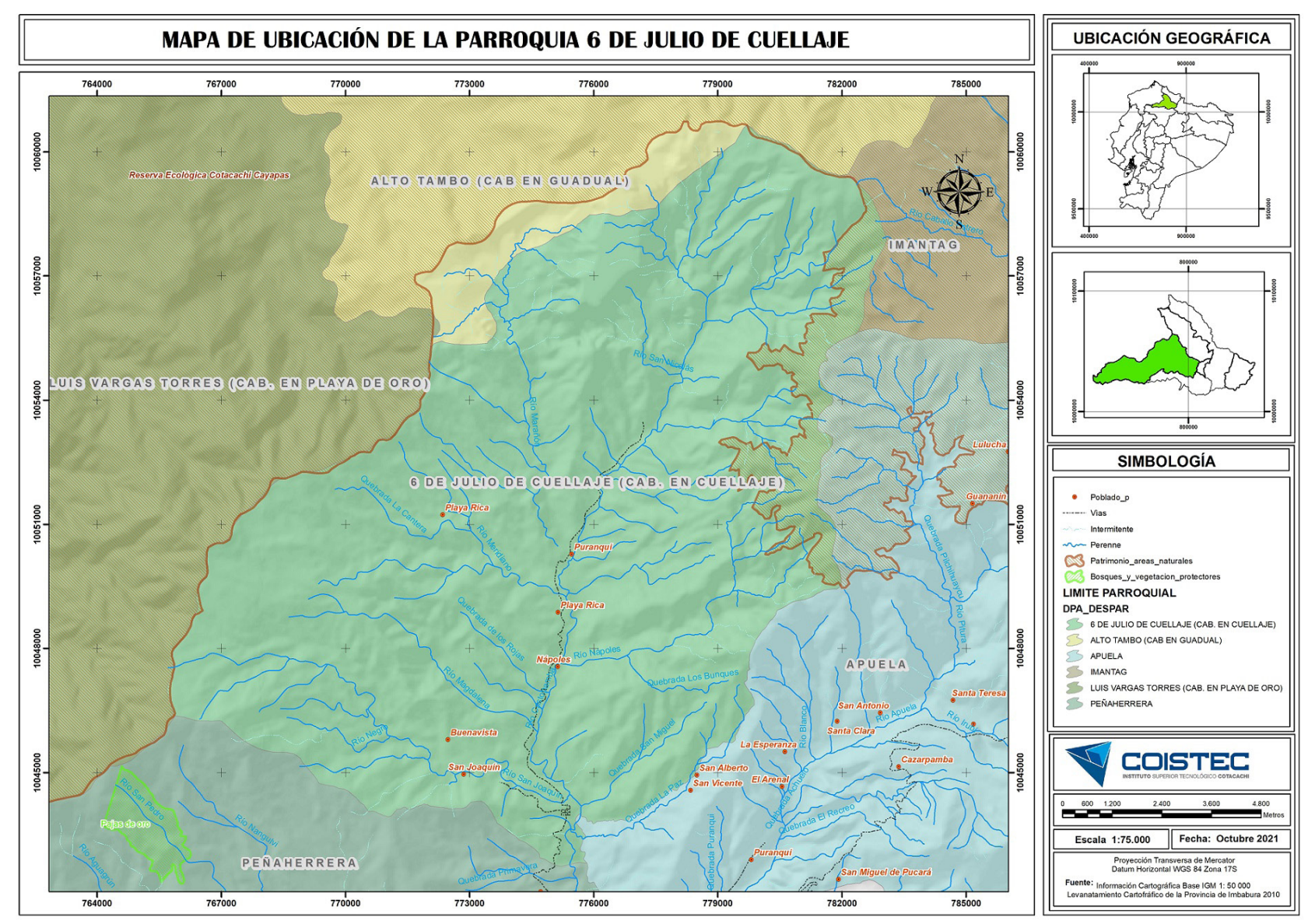

Figura 1. Mapa base de la parroquia Cuellaje.

Figure 1. Base map from parish of Cuellaje.

Nacional Cotacachi-Cayapas y la Cordillera de Toisán; cuenta con varios microclimas y diversidad de flora y fauna. La mayor área de uso de suelo está destinada a la conservación y protección (63,45\%), y en menor grado a las actividades pecuarias $(21,07 \%)$ (Gobierno Autónomo Descentralizado Parroquial de Cuellaje [GADPC], 2019).

El rango altitudinal va desde los $1.720 \mathrm{~m} \mathrm{s.} \mathrm{n.} \mathrm{m.} \mathrm{al} \mathrm{sur,} \mathrm{en} \mathrm{la} \mathrm{confluencia} \mathrm{del} \mathrm{río} \mathrm{Cristopamba} \mathrm{y} \mathrm{quebra-}$ da La Primavera, a los 3.480 m s. n. m. en la naciente del río Cristopamba (Figura 1). Presenta pendientes muy fuertes en un poco más del $80 \%$ del área. Limita al norte con la parroquia Alto Tambo del cantón San Lorenzo, al sur con la parroquia Peñaherrera del cantón Cotacachi, al este con la parroquia Imantag y Apuela del cantón Cotacachi, y al oeste con la parroquia Alto Tambo del cantón San Lorenzo y parroquia Luis Vargas Torres del cantón Eloy Alfaro.

\subsection{Elaboración del inventario de atractivos turísticos}

Se lo realiza mediante la «Metodología de jerarquización de atractivos y generación de espacios turísticos» (MINTUR, 2018), misma que se divide en tres fases. La Fase I, destinada al levantamiento y registro, inicia con la identificación de los recursos naturales y culturales presentes en el área de estudio mediante investigación primaria y secundaria. Los datos se registran en una ficha en formato Excel (XLS) diseñada por el MINTUR, donde se debe seleccionar las casillas de acuerdo con las características presentes en los atractivos.

La Fase II, corresponde a la ponderación y jerarquización. Una vez registrados los datos de los recursos y/o atractivos en la ficha XLS, automáticamente se genera una hoja de resumen con la puntuación de la ponderación que el recurso y/o atractivo obtuvo en cada uno de los criterios. Los criterios se dividen en dos grupos. El primero relacionado con el índice de competitividad turística; y el segundo, concerniente a la demanda. La suma total de los valores expresados en cada uno de los criterios es 100, como se muestra en la Tabla 2. 
Tabla 2. Criterios de valoración.

Table 2. Valuation criteria.

\begin{tabular}{lclc}
\hline \multicolumn{1}{c}{$\begin{array}{c}\text { Criterios relacionados con el índice de } \\
\text { competitividad turística }\end{array}$} & Valoración & $\begin{array}{c}\text { Criterios relacionados } \\
\text { con la demanda }\end{array}$ & Valoración \\
\hline Accesibilidad y conectividad & 18 & Difusión del atractivo & 7 \\
Planta turística / servicios & 18 & Tipo de visitante & 5 \\
Estado de conservación & 14 & Recursos humanos & 5 \\
Higiene y seguridad turística & 14 & & \\
Políticas y regulaciones & 10 & \\
Actividades que se practican en el atractivo & 9 & \\
\hline \multicolumn{2}{c}{ Suma total de valores de criterios: 100 } \\
\hline
\end{tabular}

Nota: tomado de la «Metodología de jerarquización de atractivos y generación de espacios turísticos 2018» del Ministerio de Turismo del Ecuador.

La suma de la ponderación de los nueve criterios permite determinar la jerarquía del recurso y/o atractivo. Los valores de los rangos de jerarquía se muestran en la Tabla 3.

Tabla 3. Jerarquización de atractivos turísticos.

Table 3. Ranking of tourist attractions.

\begin{tabular}{clc}
\hline Jerarquía & Descripción & $\begin{array}{c}\text { Rangos de } \\
\text { jerarquía }\end{array}$ \\
\hline IV & $\begin{array}{l}\text { Atractivo excepcional y de alta significación para el mercado turístico internacional, capaz } \\
\text { por sí solo de motivar una importante corriente de visitantes (actual o potencial). }\end{array}$ & $86-100$ \\
& Atractivo con rasgos excepcionales, capaz de motivar por sí solo o en conjunto con otros \\
atractivos contiguos, una corriente actual o potencial de visitantes nacionales o extranjeros. & $61-85$ \\
II & $\begin{array}{l}\text { Atractivo con algún rasgo llamativo, capaz de interesar a visitantes que hubiesen llegado a la } \\
\text { zona por otras motivaciones turísticas, o de motivar corrientes turísticas nacionales. }\end{array}$ & $36-60$ \\
& Atractivo sin mérito suficiente para considerarlo al nivel de las jerarquías anteriores, pero \\
que igualmente forma parte del inventario de atractivos turísticos como elemento que com- & $11-35$ \\
plementa a otros de mayor jerarquía. & $\begin{array}{l}\text { Es un elemento natural o cultural que puede motivar el desplazamiento, pero no se encuentra to- } \\
\text { davía incorporado en la dinámica turística, ni cuenta con ningún tipo de infraestructura de apoyo. }\end{array}$ & $0-10$ \\
\hline
\end{tabular}

Nota: tomado de la «Metodología de jerarquización de atractivos y generación de espacios turísticos 2018» del Ministerio de Turismo del Ecuador.

\subsection{Valoración de potencial turístico}

Los criterios de valoración del MINTUR tienen una ponderación estándar, por lo que se requiere determinar el porcentaje de cumplimiento alcanzado por el recurso y/o atractivo en cada criterio de valoración. Para ello, se adapta la ecuación [1] utilizada por Bravo Peña et al. (2015).

$$
\% \text { de cumplimiento } \frac{\text { Resultado de valores de ponderación de atractivo }}{\text { Ponderación estándar MINTUR }} * 100
$$

Los resultados obtenidos en la ponderación de los nueve criterios de valoración (Tabla 2), se compilan en una matriz de doble entrada; en las filas se sitúan los criterios de valoración y la ponderación estándar, y en las columnas se ubican los nombres de los recursos y la ponderación que obtuvieron en cada criterio.

Posteriormente, se suman las ponderaciones que los recursos y/o atractivos obtuvieron en cada uno de los criterios de valoración, lo que permite observar de manera global el porcentaje de cumplimiento de los criterios de valoración a nivel de territorio.

El nivel de potencialidad turística se midió en tres rangos: alto, medio y bajo. Se sumaron las ponderaciones de los criterios de valoración de 20 atractivos turísticos de la parroquia Seis de Julio de Cuellaje. La puntuación de cada rango se indica en la Tabla 4. 
Tabla 4. Niveles de potencialidad turística.

Table 4. Levels of tourist potentiality.

\begin{tabular}{cc}
\hline Nivel de potencialidad & Porcentaje de cumplimiento/ponderación estándar \\
\hline Alto & $65-100$ \\
Medio & $35-65$ \\
Bajo & $<35$ \\
\hline
\end{tabular}

Nota: adaptación de Reyes Pérez y Sánchez Crispín (2015).

El análisis de los criterios se realiza de mayor a menor porcentaje, contrastando las condiciones del criterio con la realidad de la parroquia como destino turístico emergente.

\section{Resultados}

\subsection{Inventario de atractivos turísticos}

Los resultados de la ponderación y jerarquización de 20 atractivos turísticos de la parroquia Seis de Julio de Cuellaje se presentan en la Tabla 5.

Tabla 5. Ponderación y jerarquización de los atractivos turísticos.

Table 5. Weighting and ranking of tourist attractions.

\begin{tabular}{lllc}
\hline Nombre del Atractivo & Típo & $\begin{array}{c}\text { Resultado de } \\
\text { ponderación }\end{array}$ & Jerarquía \\
\hline Bosque de alisos & Bosques & 26,5 & I \\
Bosque nublado San Antonio & Bosques & 28,0 & I \\
Cascada San Joaquín & Ríos & 30,5 & I \\
Cascada Rosario & Ríos & 29,0 & I \\
Cascada San Alberto & Ríos & 25,5 & I \\
Cascada San Antonio & Ríos & 23,5 & I \\
Mirador La Loma & Montañas & 31,5 & I \\
Río Cristopamba & Ríos & 33,5 & I \\
Río Negro San Joaquín & Ríos & 33,5 & I \\
Río San Joaquín & Ríos & 33,5 & I \\
Vado Don Jeremías & Ambientes lacustres & 30,5 & I \\
Vado La Unión & Ambientes lacustres & 30,5 & I \\
Vado Nápoles & Ambientes lacustres & 33,5 & I \\
Fiesta del Día de los Difuntos & Acervo cultural y popular & 37,0 & II \\
Fiesta de la Virgen de El Quinche & Acervo cultural y popular & 40,5 & II \\
Fiesta de parroquialización & Acervo cultural y popular & 39,0 & II \\
Fiesta de Fin de Año & Acervo cultural y popular & 35,0 & I \\
Molienda de caña Magdalena & Realizaciones técnicas y científicas & 25,5 & I \\
Piscina de truchas San Joaquín & Realizaciones técnicas y científicas & 30,5 & 34,5 \\
Vestigios arqueológicos San Joaquín & Arquitectura & & I \\
\hline
\end{tabular}

El $65 \%$ (13) de los atractivos identificados corresponden a atractivos naturales y el $35 \%$ (7) a manifestaciones culturales. Diecisiete atractivos alcanzaron la jerarquía I destacándose los sitios naturales relacionados con el recurso agua, representados por ríos y cascadas. En jerarquía II se ubican tres atractivos vinculados a manifestaciones culturales, expuestos en las fiestas tradicionales que se realizan en la localidad con periodicidad anual. En la Figura 2 se muestra la distribución geográfica de los atractivos turísticos identificados en el territorio. 


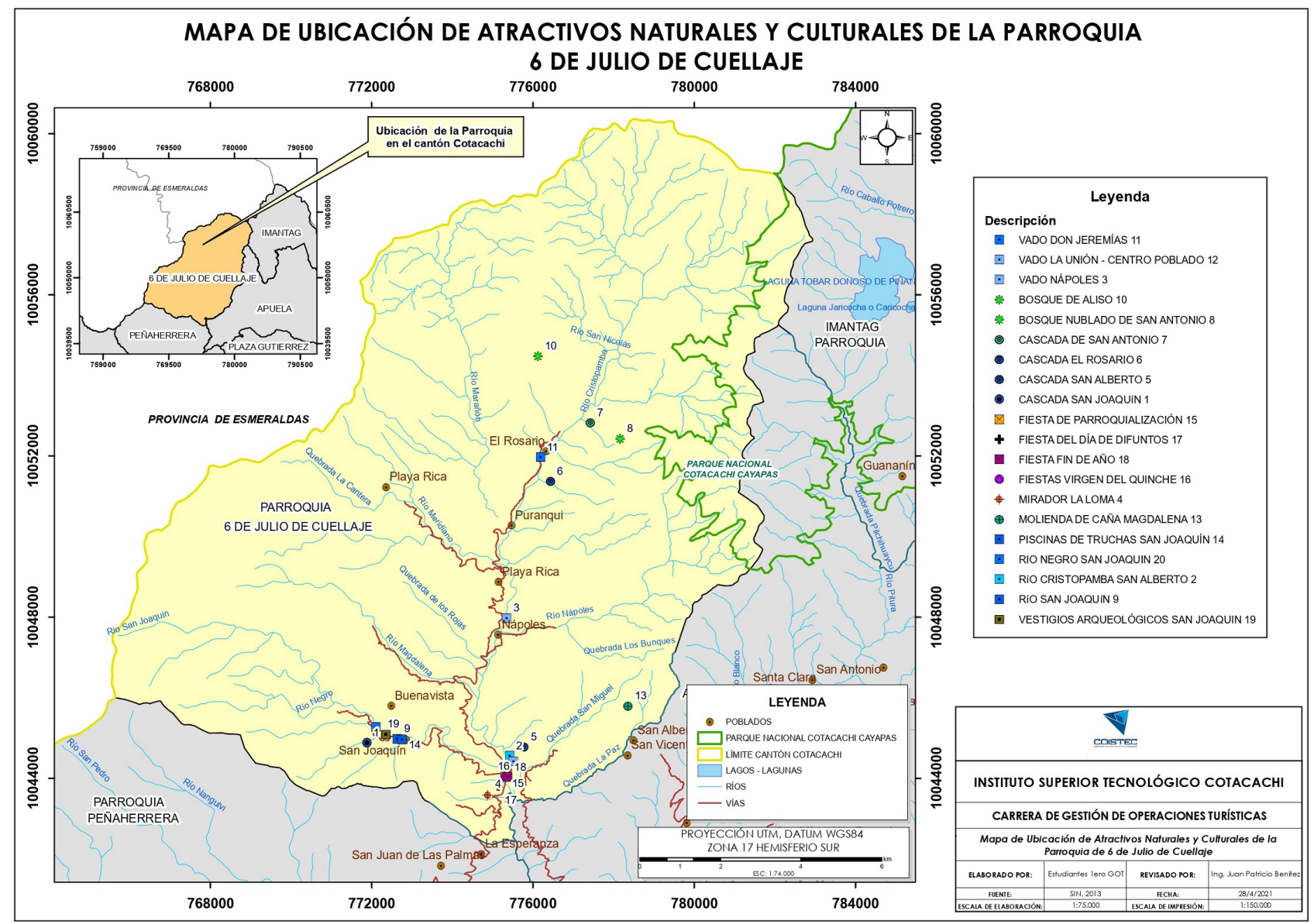

Figura 2. Mapa de atractivos turísticos naturales y culturales.

Figure 2. Map of natural and cultural tourist attractions.

Se aprecia que la distribución espacial de los atractivos turísticos es diversa, puesto que algunos atractivos se encuentran cerca de la cabecera parroquial y otros distantes. La ubicación geográfica incide en la jerarquización de los atractivos (MINTUR, 2018). Los atractivos de jerarquía II, presentan rasgos llamativos y condiciones básicas para la generación de productos turísticos capaces de interesar a visitantes que hubiesen llegado a la zona por otras motivaciones; en el caso de Cuellaje se asocian a las fiestas locales. Los atractivos de jerarquía I se encuentran alejados del centro poblado, por lo que carecen de servicios básicos y turísticos complementarios. Aún no presentan condiciones mínimas necesarias para generar productos turísticos.

\subsection{Valoración del potencial turístico}

La determinación del porcentaje de cumplimiento alcanzado por el recurso y/o atractivo en cada criterio de valoración se resume en la Tabla 6.

La valoración del criterio, estado de conservación e integración sitio/entorno, alcanzó el 61,4% de la ponderación estándar. La conservación responde a la estimación de la integridad de los atributos físico-ambientales y socioculturales, con relación a las condiciones del atractivo y su entorno (MINTUR, 2018). El territorio en estudio está localizado en las estribaciones de la Cordillera de los Andes. La orografía es accidentada con presencia de bosques primarios y secundarios. Los ecosistemas conservan la riqueza natural y paisajística por la escasa intervención de los lugareños. La conservación de los atractivos motiva la visita de turistas que buscan reencontrarse con la naturaleza.

La accesibilidad y conectividad alcanzaron el 35,6 \%. Es decir, los atractivos turísticos no son accesibles para personas con discapacidad (MINTUR, 2018). En cuanto a la conectividad, la principal vía de acceso a la parroquia desde la cabecera cantonal es de primer orden. La red vial interna es de segundo y tercer orden con una longitud aproximada de 52,6 km. En época de invierno el ingreso a la parroquia se dificulta debido a pequeños deslizamientos de tierra y formación de charcas. El mantenimiento de las vías que integran esta red se realiza en coordinación entre los GAD locales y el trabajo ejecutado a través de 
Tabla 6. Valoración del potencial turístico.

Table 6. Tourist potential assessment.

\begin{tabular}{lcc}
\hline Criterios de valoración & Ponderación alcanzada & Nivel de Potencialidad \\
\hline Accesibilidad y conectividad & $35,6 \%$ & Media \\
Planta turística/ servicios & $8,3 \%$ & Baja \\
Estado de conservación e integración sitio/entorno & $61,4 \%$ & Media \\
Higiene y seguridad turística & $22,2 \%$ & Baja \\
Políticas y regulaciones & $11,1 \%$ & Baja \\
Actividades que se practican en el atractivo & $19,2 \%$ & Baja \\
Difusión del atractivo & $1,7 \%$ & Baja \\
Tipo de visitante y afluencia & $11,1 \%$ & Baja \\
Recursos Humanos & $0,0 \%$ & Baja \\
\hline
\end{tabular}

mingas comunitarias. El mejoramiento de la vialidad genera beneficios directos para un número importante de consumidores y promueve, de forma indirecta, la actividad turística en las regiones y mercados que se conectan a través de sus rutas (Lichtle Fragoso et al., 2015).

La higiene y seguridad turística de los atractivos presentan limitaciones, obteniendo el 22,2\%. Los indicadores de este criterio de acuerdo con el MINTUR (2018) son: gestión de dispositivos para la recolección de basura, procedencia del agua, presencia de actos vandálicos, limpieza e iluminación de los atractivos turísticos. Actualmente, el $100 \%$ de viviendas de la cabecera parroquial disponen del servicio de recolección de basura; existe una Unidad de Policía Comunitaria que resguarda la seguridad del poblado; más del $50 \%$ de la población no se beneficia de la red pública de agua potable y no se registran actos vandálicos (GADPC, 2019). Los servicios de higiene y seguridad turística se localizan en la cabecera parroquial, mientras que los atractivos se ubican en las comunidades; esto explica la baja calificación del criterio. La higiene y seguridad en los atractivos es primordial, considerando que la calidad del agua garantiza la prevención de enfermedades en el visitante; la recolección de basura evita la contaminación ambiental y visual del atractivo, y la presencia de la Policía Nacional impide que se cometan actos vandálicos (Soto Delgadillo, 2019).

Las actividades que se practican en el atractivo alcanzaron el 19,2\%. El porcentaje es relativamente bajo, considerando que la zona cuenta con varios atractivos naturales y culturales potenciales para el desarrollo de actividades turísticas, mismas que son un factor clave para la elección de un destino turístico (Sánchez Cuenca et al., 2020). Las actividades que se practican en el atractivo deben presentarse como un producto que motive la visita turística (Sánchez Cuenca et al., 2020). Esta tarea en la zona de estudio aún no se ha desarrollado a pesar de que la parroquia Cuellaje cuenta con escenarios propicios para la práctica de actividades en el agua y el espacio terrestre (GADPC, 2019).

En los atractivos turísticos identificados en la zona de estudio no existe un reporte estadístico de visitas. No obstante, informantes claves proporcionan datos que deben ser sujetos a validación. Este criterio obtuvo $11,1 \%$. Cabe aclarar que registrar la visita turística de un destino posibilita identificar las características del turista con el propósito de orientar la oferta a sus expectativas, necesidades y objetivos; también incide en el establecimiento de acciones de publicidad y comercialización en los segmentos de interés (Garcia Reinoso et al., 2020).

Las políticas y regulaciones son deficientes en la parroquia (11,1\%). El GADPC actualmente no cuenta con un plan de desarrollo turístico. Si bien la parroquia está incluida en el Plan de Desarrollo Turístico Cantonal, hasta el momento no se han ejecutado acciones relevantes en favor del turismo (E. Varela, comunicación personal, 16 de julio de 2021). Los GAD cantonales de acuerdo con el art. 240 de la Constitución de la República del Ecuador (2008) tienen la competencia para dictar normas de aplicación general y obligatoria dentro de su jurisdicción. Existe normativa cantonal que regula las actividades turísticas, sin embargo, la mayor parte de los establecimientos de la zona de estudio operan de manera irregular (A. Flores, comunicación personal, 18 de julio de 2021). El desarrollo turístico de un territorio implica el trabajo mancomunado de los distintos actores públicos, privados y comunitarios, que mediante la coordinación de acciones generen planes para el desarrollo turístico en el territorio (Vasallo Villalonga et al., 2019). 
La planta turística y servicios complementarios son escasos en la zona de estudio (8,3\%). El destino turístico debe estar preparado para facilitar la permanencia del visitante y satisfacer las necesidades de alimentación, hospedaje, recreación, desplazamiento, entre otros (Renda y Teotónio, 2017). Los servicios en conjunto se denominan planta turística (MINTUR, 2018). Actualmente, Cuellaje cuenta con un establecimiento de alojamiento en la categoría refugio, mientras que en el área de alimentos y bebidas los negocios existentes no se encuentran registrados por la autoridad nacional competente. La promoción y comercialización de los atractivos registrados en el territorio de Cuellaje es incipiente (1,7 \%). Los atractivos no se encuentran incluidos en paquetes turísticos y tampoco son ofertados por operadoras locales (E. Varela, comunicación personal, 16 de julio de 2021). Ningún atractivo turístico inventariado cuenta con talento humano certificado (0\%). El talento humano es un componente determinante para el quehacer turístico, puesto que el profesional forma parte del producto turístico (Kotler et al., 2015).

Finalmente, se determina que los atractivos turísticos de la parroquia de Cuellaje tienen un nivel bajo de potencialidad turística en el $78 \%$ de los criterios de valoración. El porcentaje restante $(22 \%)$ presenta una potencialidad media y se relaciona con el estado de conservación y la accesibilidad y conectividad.

\section{Discusión}

De las cualidades que se pueden destacar del método propuesto están el número de criterios que analiza (seis relacionados con el índice de competitividad y tres con la demanda). La ponderación de los criterios dio como resultado tres atractivos turísticos de jerarquía II situados cerca del centro poblado y 17 de jerarquía I dispersos en el territorio rural. Los criterios de valoración empleados en el estudio integran características fundamentales que debe tener un método de evaluación de potencialidad del territorio como lo explican Reyes Pérez y Sánchez Crispín (2015).

Se reveló que el nivel de potencialidad más alto en la parroquia en estudio está dado por los criterios: estado de conservación sitio/entorno y accesibilidad y conectividad. Estos resultados son similares a los obtenidos por Sánchez Cuenca et al. (2020) y Lazo Serrano et al. (2017).

En la metodología del MINTUR, se evalúa los atractivos naturales y culturales por jerarquías. La jerarquía de cada atractivo representa su importancia dentro de su propia categoría, pero no establece un nivel de potencialidad específico que presenta el territorio. Para determinar la potencialidad es necesario establecer una ponderación que diferencie aquellos criterios que satisfacen las necesidades de los turistas, de los que carecen de estas cualidades (Reyes Pérez y Sánchez Crispín, 2015).

De las debilidades del método se deja en discusión que los resultados dependen estrechamente de las ponderaciones en la valoración de criterios, por lo que se debe prestar atención a los datos recolectados in situ y la digitación en la ficha xls, así lo corroboran Garzón García y Florido Trujillo (2021). De igual modo, es un obstáculo que la ficha no cuente con suficiente información de la realidad socioeconómica, la dimensión natural, como índices de biodiversidad, distribución de vida silvestre, valor ecológico, resiliencia ambiental, por nombrar algunos ejemplos usados en otros trabajos como el de Mikery Gutiérrez y Pérez-Vázquez (2018), mismos que permiten mayor precisión en la determinación de la potencialidad turística de un territorio.

Aunque en la mayoría de criterios de valoración la potencialidad es baja dada la condición geográfica del territorio rural, esta misma característica le proporciona al espacio potencialidad para realizar distintas actividades turísticas como camping, ciclismo, rappel, cabalgata, observación de flora y fauna, entre otras (Nájera González et al., 2021). Determinar el potencial turístico del territorio rural implica integrar la perspectiva de los diferentes actores que se encuentran relacionados con el territorio y el sector turístico. Por ello, es necesario integrar las perspectivas de los diferentes conocimientos, tanto del experto, del residente y del visitante como de las autoridades locales que gestionan los diferentes programas para el desarrollo turístico local (Mikery Gutiérrez y Pérez-Vázquez, 2018).

Al igual que Cuellaje otros territorios del país presentan atractivos turísticos conservados, pero no tienen el soporte de infraestructura turística, servicios complementarios, talento humano, entre otros factores para desarrollar el turismo. Un ejemplo de este hecho es el estudio realizado en las parroquias rurales del cantón Santa Rosa en la provincia de El Oro, Ecuador (Sánchez Cuenca et al., 2020), donde la única fortaleza identificada es el criterio inherente a la conservación del atractivo. 


\section{Conclusiones}

Mediante la aplicación de la metodología para la jerarquización de atractivos y generación de espacios turísticos del MINTUR (2018) se identificaron en el área de estudio 20 atractivos de jerarquía I y II, localizados principalmente en zonas montañosas circundantes a sistemas hídricos que actualmente se encuentran conservados. Este estudio contribuye a la planificación turística de la parroquia Seis de Julio de Cuellaje con el registro técnico de sitios con características singulares que pueden ser considerados luego de la valoración de criterios de ponderación como atractivos turísticos.

La ponderación de los nueve criterios de valoración estableció que los criterios con potencialidad media son: conservación e integración atractivo/entorno con $61,4 \%$ y la accesibilidad y conectividad con $35,6 \%$. La potencialidad del territorio es baja, en relación con los demás criterios. La accesibilidad, conectividad y planta turística son escasos en los atractivos, reflejando una situación de existencia, aunque con algunas limitaciones para la prestación de servicios turísticos. Lo mismo ocurre con las políticas y regulaciones que denotan la ausencia de planificación turística local. Por otro lado, la difusión del atractivo es casi nula y, por ende, existe escasa afluencia de visitantes a este sector. A ello se suma la ausencia de recurso humano calificado para la prestación de servicios turísticos. En consecuencia, el territorio aún no presenta las condiciones necesarias para la práctica de actividades turísticas y la satisfacción de necesidades de los visitantes. Si bien es cierto, hay atractivos que llaman la atención, el sistema turístico es incipiente.

Finalmente, la presente investigación constituye un primer insumo para el diagnóstico turístico local de la parroquia Seis de Julio de Cuellaje. Cabe recalcar que el inventario previo a la inclusión en la planificación turística local debe ser validado por la autoridad nacional de turismo. Además, es necesaria la concertación público-privada, a nivel local/regional para la generación de productos turísticos que influyan en la decisión de visita.

\section{Agradecimientos}

Los autores agradecen a la Doctora Natalia Doukh, Rectora del Instituto Tecnológico Superior Cotacachi (COISTEC) y al Magíster Ismael Yépez, Coordinador de Investigación y Desarrollo Tecnológico del COISTEC por todas las facilidades brindadas para el desarrollo del trabajo de investigación. También se reconoce el involucramiento de los estudiantes de la Tecnología Superior en Gestión de Operaciones Turísticas en el levantamiento y sistematización de información.

\section{Contribuciones de los autores}

- Lorena Elizabeth Casanova Imbaquingo: conceptualización, investigación, metodología, redacción revisión y edición.

- Tatiana Elizabeth Guerrero Cabezas: investigación, redacción - borrador original.

- Juan Patricio Benítez Pereira: supervisión, visualización, investigación.

\section{Referencias}

Banco Central del Ecuador. (2017). Cuentas nacionales regionales. Cuentas provinciales (anuales). https:// www.bce.fin.ec/index.php/component/k2/item/293-cuentas-provinciales

Benavides Vindas, S. (2019). El aporte del turismo a la economía costarricense: más de una década después. Economía y Sociedad, 25(57), 1-29. https://doi.org/10.15359/eys.25-57.1

Bravo Peña, L. C., Sáenz López, D. A., Alatorre Cejudo, L C., Priego Santander, A., Torres Olave, M. E., y Granados Olivas, A. (2015). Identificación de áreas potenciales de recarga hídrica al acuifero Cuauhtémoc (Chihuahua), mediante una evaluación espacial multicriterio. Memorias de resúmenes en extenso SELPER-XXI. Ciudad Juárez, Chihuahua, México. http://www3.uacj.mx/CGTI/CDTE/JPM/Documents/SELPER/assets/et023.pdf

Camara, C. J., Gómez Ortega, G., y Morcate Labrada, F. (2019). Instrumental para evaluar los recursos territoriales turísticos del centro de ciudad de Fort-de- France. Revista Cientifica de Arquitectura y Urbanismo, 40(1), 52-71. https://rau.cujae.edu.cu/index.php/revistaau/article/view/518 
Constitución de la República del Ecuador [Const]. 20 de octubre de 2008.

Dávalos, X., Pinargote, M., y Brucil, G. (2019). Una mirada desde el turismo sustentable a la provincia de Imbabura - Ecuador. Espacios, 40(43), 12. https://www.revistaespacios.com/a19v40n43/19404312.html

Flores Rodríguez, O., Morales Ramírez, D., y Moreno Velazco, F. (2019). Evaluación del potencial turístico de una ciudad ubicada en la cuenca de Burgos, Tamaulipas. Revista Turydes: Turismo y Desarrollo, 12(27). https://www.eumed.net/rev/turydes/27/turismo-tamaulipas.html

Garcia Reinoso, N., García Moreira, D. y Quintero Ichazo, Y. (2020). Segmentación de la demanda turística colombiana que visita la Zona de Planificación 1 más la provincia de Pichincha en Ecuador. Revista Interamericana de Ambiente y Turismo, 16(2), 136-152. https://riat.utalca.cl/index.php/test/article/view/564

Garzón García, R., y Florido Trujillo, G. (2021). Nuevos planteamientos en la docencia universitaria en turismo: propuesta didáctica para el estudio de los recursos territoriales turísticos. Cuadernos de Turismo, (47), 329-363. https://doi.org/10.6018/turismo.474141

Gobierno Autónomo Descentralizado Parroquial de Cuellaje [GADPC]. (2019). Plan de Desarrollo y Ordenamiento Territorial de Cuellaje. GADPC. https://www.cuellaje.gob.ec/images/PDOT_CUELLAJE_2019-2023.pdf

Kotler, P., Bowen, J. T., y Makens, J. C. (2015). Marketing turístico (6 ${ }^{\mathrm{a}}$ ed.). Pearson.

Lazo Serrano, C. A., Bastidas, M. I., Aguilar, F. E., y Calle, M. P. (2017). La potencialidad turística y sus oportunidades de emprendimiento. Caso Pasaje. INNOVA Research Journal, 2(8.1), 1-21. https://doi. org/10.33890/innova.v2.n8.1.2017.320

Lichtle Fragoso, P. M., Sánchez Salinas, J. C., López Noría, D. A., y Padilla Jiménez, J. A. (2015). Evolución de la conectividad en la red aérea mexicana y el crecimiento del turismo nacional. Documentos de Investigación Estadística y Económica, No. 2015-2. Secretaría de Turismo.

Marzo-Navarro, M., Pedraja-Iglesias, M., y Vinzón, L. (2017). Key variables for developing integrated rural tourism. Tourism Geographies, 19(4), 575-594. https://doi.org/10.1080/14616688.2017.1336785

Mikery Gutiérrez, M. J., y Pérez-Vázquez, A. (2018). Métodos para el análisis del potencial turístico del territorio rural. Revista Mexicana de Ciencias Agricolas, (9), 1729-1740. https://doi.org/10.29312/ remexca.v0i9.1060

Ministerio de Turismo del Ecuador [MINTUR]. (2018). Manual de atractivos turísticos. MINTUR.

Ministerio de Turismo del Ecuador [MINTUR]. (2019). Plan Nacional de Turismo 2030. MINTUR. https:// www.turismo.gob.ec/wp-content/uploads/2020/03/PLAN-NACIONAL-DE-TURISMO-2030-v.-final-Registro-Oficial-sumillado-comprimido_compressed.pdf

Ministerio de Turismo del Ecuador [MINTUR]. (2021). Cuenta Satélite de Turismo. Indicadores económicos del turismo. https://servicios.turismo.gob.ec/index.php/18-turismo-en-cifras/analisis-economico/97-cuenta-satelite-de-turismo

Nájera González, A., Carrillo González, F., Chávez Dagostino, R., y Nájera González, O. (2021). Proceso metodológico de evaluación de la aptitud del territorio para actividades de turismo alternativo: caso de estudio Miramar-Playa Tortugas, Riviera Nayarit, México. Investigaciones Turísticas, (21), 256-277. https:// doi.org/10.14198/INTURI2021.21.12

Renda, A. I., y Teotónio, I. (2017). Alojamiento turístico en espacio rural. La percepción de los emprendedores. Estudios y perspectivas del turismo, 26(4), 845-864. https://www.estudiosenturismo.com.ar/PDF/V26/ N04/v26n4a05\%20.pdf

Reyes Pérez, Ó., y Sánchez Crispín, Á. (2005). Metodología para determinar el potencial de los recursos turísticos naturales en el estado de Oaxaca, México. Cuadernos de Turismo, (16), 153-174. https://revistas.um.es/turismo/article/view/18341

Sánchez Cuenca, L. M., Martínez Romero, D. A., y Parra Loayza, D. C. (2020). Determinación del producto turístico basado en ecoturismo por medio de la potencialidad: Caso Parroquias Rurales de Santa Rosa, El Oro, Ecuador. Turismo, Desarrollo y Buen Vivir. Revista de Investigación de la Ciencia Turística - RICIT, (14), 7-23. https://publicaciones.udet.edu.ec/index.php/ricit/article/view/25

Sánchez Zhingri, K. B., Troya Zúñiga, C. E., y Calle Iñiguez, M. P. (2020). Análisis de la Potencialidad Turística de las parroquias Uzhcurrumi y Casacay del cantón Pasaje, El Oro, Ecuador. Kalpana - Revista de Investigación, (18), 33-52. https://publicaciones.udet.edu.ec/index.php/kalpana/article/view/9

Segura Ronquillo, S. (2015). El aporte de la actividad turística y el ingreso de divisas para el Ecuador. Revista Empresarial, ICE-FEE-UCSG, 9(3), 35-43. https://editorial.ucsg.edu.ec/ojs-empresarial/index.php/empresarial-ucsg/article/view/23 
Soto Delgadillo, J. M. (2019). Seguridad en el destino turístico, específicamente en la ciudad de La Habana, Cuba, y en el centro histórico de Bogotá. Papeles de Administración Hoy, 09(13), 74-79. http://repository. unipiloto.edu.co/handle/20.500.12277/10369

Toselli, C. (2019). Turismo, patrimonio cultural y desarrollo local. Evaluación del potencial turístico de aldeas rurales en la provincia de Entre Ríos, Argentina. PASOS Revista de Turismo y Patrimonio Cultural, 17(2), 343-361. https://doi.org/10.25145/j.pasos.2019.17.024

Vasallo Villalonga, Y., Rhea González, B. S., y La Serna Gómez, A. M. (2019). La planificación y competitividad turística ecuatoriana en el contexto del geoparque Imbabura. Explorador Digital, 3(3), 15-25. https:// doi.org/10.33262/exploradordigital.v3i3.440 